\title{
El sistema medieval de asentamientos en Castilla y León
}

\author{
Félix Benito Martín *
}

\begin{abstract}
RESUMEN
Éste título obedece al intento de formular cómo se produjo el sistema de asentamientos en el actual territorio de Castilla y León. Sobre un terreno virgen, despoblado a raíz del control musulmán de la península, hasta que a partir del siglo $X$ se produce un intenso fenómeno de creación urbana, semejante pero más comprimido en el tiempo que en resto de Europa. Se indican diferentes periodos de asentamiento sobre este terreno despoblado: sistema altomedieval, evolución plenomedieval al norte del Duero, la repoblación entre diversas líneas fronterizas entre reinos cristianos y la colonización de las tierras al sur del reino tras la toma de Toledo por Alfonso VI en 1085. Este intenso proceso repoblador, de gran trascendencia en el control del territorio y finalmente en el dominio de gran parte de la península, fue también el antecedente de la tarea de colonización del continente americano, que Castilla realiza con una enorme eficacia en un periodo de treinta años del siglo XVI.
\end{abstract}

PALABRAS CLAVE: Historia de la ciudad; territorio y sistema de asentamientos; formación urbana en Castilla y León; Comunidades de Villa y Tierra.

En este artículo se resume la formación del sistema de asentamientos de los reinos cristianos en la Edad Media, correspondientes a la actual región de Castilla y León, durante los siglos de formación de la red urbana entre finales del siglo IX y comienzos del siglo XIII: en esta época se forma el sistema de asentamientos que configurará la red urbana que permanecerá durante un milenio y que será la estructura del sistema urbano actual de la región, como ocurre

\begin{abstract}
This heading goes according with the aim of making out how was it produce the organization of the population centres in the actual territory of Castilla y León. On virgin places, inhabited because of the Moslem control on the Iberian Peninsula, from the $X$ century an intensive phenomenon of urban creation appeared, similar but shorter than in the rest of Europe. They are shown different urban centres periods on these unpopulated areas: high-medieval system, plenum medieval evolution on the north of the Duero River, the repopulation of different Christian kingdoms and the colonization of the south places after the Toledo's conquest by Alfonso VI in 1085.

This intensive repopulation process, so transcendental in the control of the territories and finally in the dominance of a big part of the Peninsula, was also the antecedent of the colonization job of the American Continent, that Castilla realized with a huge efficiency on a thirty year period of the XVI century.
\end{abstract}

KEY WORDS: Historia de la ciudad; territorio y sistema de asentamientos; formación urbana en Castilla y León; Comunidades de Villa y Tierra.

en el resto de Europa y del mundo antiguo, que incluye territorios de África y de Asia.

Los reinos cristianos realizan su avance de repoblación en la meseta norte desde el siglo IX hasta la conquista de Toledo en 1085. Durante este periodo de más de dos siglos se producen diversos modos de repoblación. En una primera etapa la repoblación de los cristianos del norte alcanza hasta el Duero, momento en

* Catedrático de Urbanismo y Ordenación del Territorio. Escuela Superior de Arte y Arquitectura. Universidad Europea de Madrid. 
que se fija la frontera en este río con las posiciones de Zamora, Toro, Simancas y Aranda. Al otro lado de la frontera los terrenos pertenecen al califato de Códoba, más culto y mucho más poderoso militarmente, pero que ternía menos interés en repoblar la meseta. Así la zona entre el Duero y el Tajo permanecía prácticamente desierta, eso sí con el poderoso control militar del califato.

Al norte del Duero se produce durante los siglos $I X$ y $X$ un proceso de repoblación altomedieval, que más tarde se transformará durante los siglos XI y XII hasta convertirse en las áreas centrales en un esquema en el que los núcleos responden a recintos murados, especialmente en la áreas centrales de la meseta. A estos procesos de doble y paulatina generación se contrapone todo el esfuerzo de control del territorio plasmado al sur del Duero tras la conquista de Toledo en 1085, y ampliación de la frontera hasta el Tajo. Entre este río y el Duero se utiliza el método de las comunidades de Villa y Tierra, donde se concentran los mecanismos urbanos y de control en determinadas ciudades o cabezas de comunidad, siendo estas villas las que organizan y controlan todo el territorio y la gran cantidad de aldeas sin muralla de cada entidad.

El panorama se completa con las villas fronterizas que se fundan sobre todo para garantizar la referencia y control de esas zonas y la fidelidad al reino promotor de su fundación. Curiosamente son las fronteras entre reinos cristianos las que más han sido objeto de este reforzamiento mediante la colonización. En este apartado especial importancia tuvo la división del reino por Alfonso VII entre los reinos de León y Castilla, que permaneció durante 73 años.

\section{LA ÉPOCA ALTOMEDIEVAL. 840-I035. LA REPOBLACIÓN AL NORTE DEL DUERO}

La estabilización de la frontera cristianomusulmana en la línea del Duero desde 940 hasta 1085 divide la meseta en dos zonas cuyo carácter en cuanto al sistema de asentamien- tos va a ser completamente diferente. En la mitad septentrional se fue decantando una red, que en un primer momento será indiferenciada para posteriormente estructurarse y jerarquizarse. Toda una serie de fenómenos tuvieron lugar, primero durante los siglos $X y X I$, en que se produce la primera etapa de asentamientos, para, imperceptiblemente, durante la segunda mitad de este siglo $(\mathrm{XI})$ y sobre todo durante el siguiente llegar a un sistema más complejo y articulado, en el que los núcleos urbanos empiezan a consolidarse.

Por el contrario, al sur del Duero este proceso no se produce de un modo evolutivo, sino que se crea una estructura urbana a partir de los resultados producidos en las áreas septentrionales. Este nuevo sistema se introduce debido a la necesidad de mayor eficacia y rapidez necesaria para colonizar el amplio espacio que quedaba asumido entre las líneas del Duero y del Tajo, tras la conquista de Toledo en 1085. Estas zonas se denominaron entonces Extremadura, al sur del Duero, y Transierra, al sur del Sistema Central. Aquí se estableció un sistema de comunidades de Villa y Tierra en el que una villa asumía todo el poder institucional y de control del territorio y el resto eran aldeas dependientes de la cabecera y carecían de muralla.

Hemos visto como la zona norte, profundamente desarticulada tras los sucesos del siglo VIII, había quedado extremadamente deprimida desde el punto de vista demográfico

Todo hace pensar que las estructuras territoriales visigóticas y tardo-romanas se descompusieron efectivamente. De la antigua red urbana queda el nombre y prestigio de algunos asentamientos tal como lo reflejan las crónicas del siglo $X$. Tales núcleos apenas estarían habitados, siendo a lo más pequeños puntos débilmente fortificados sobre las ruinas de ciudades anteriores.

No sólo las crónicas sino sobre todo los cartularios de los grandes monasterios, que concentrados en el tercio norte de la meseta irradiaron su potente efecto de dominio sobre el medio, nos han dejado gran cantidad de testi- 
monios sobre el tipo de asentamiento que se produjo en este período.

Tres son las denominaciones más habituales que se dan a los lugares habitados en este época: civitas, castrum y villae. Son tipos de núcleos cuyos perfiles no aparecen claramente definidos y a veces presentan una cierta ambigüedad en sus términos.

La "civitas" es una denominación común al occidente europeo y denomina a un tipo de poblaciones en las que se desarrolla un cierto poder institucional y una asecendencia sobre el territorio circundante que, en numerosas ocasiones, incluye la dignidad episcopal.

En la meseta existen poblaciones que habían sido sede episcopal durante la época visigótica (Astúrica, León, Auca, Palantia, Salmántica, Avila, Segovia y Uxama).

Junto a ellas, otras ciudades comienzan a desempeñar un papel decisivo y adquieren el carácter de "civitates", asumiendo las funciones episcopales, como sucede en el caso de Zamora.

De entre todas las poblaciones que se habían hecho acreedoras a la denominación "civitas", se consolidarán como tales durante el siglo XI algunas de ellas, cuyo carácter rector se va a manifestar a lo largo de la Edad Media e incluso épocas posteriores. Tienen en común haber alcanzado el rango de sede episcopal, lo que les garantizó una pervivencia en su papel institucional a lo largo de los siglos. León, Astorga y Zamora, en el reino de León, son las principales. Las dos primeras surgen sobre los recintos de trazado romano que aún se conservaban, la tercera es, sin embargo, una "civitas" de nueva creación. Poder militar, poder eclesiástico y dominio sobre el territorio son tres aspectos que definen el carácter de estos núcleos. Son sin duda los que presentan mayor complejidad urbana de toda la meseta en estos tiempos altpomedievales. En el caso de Astorga y León, conocemos exactamente su extensión, que corresponde con el primitivo asentamiento romano, cuyos muros se reconstruyeron en esta época. La configuración exacta de Zamo- ra es algo más incierta, pero sabemos que se trata de un núcleo de cierta importancia surgido en el extremo de un espolón vecino al Duero y cuyo trazado refleja una disposición de origen típicamente medieval.

La contextura del resto de las "civitates" es menos conocida, aunque se puede deducir que fueron de escasa magnitud y con la reducida entidad demográfica, quizá apenas suficiente para servir de base al control del territorio circundante.

El origen de la denominación "castrum" aparece muy tempranamente en las crónicas referidas a poblaciones. Ya desde el siglo VIII queda claro su carácter genuinamente militar y de control del territorio. Muchos de ellos se levantan sobre asentamientos preesxistentes ibéricos o hispano-romanos, pero en otros casos son de nueva creación. En ocasiones la existencia de un punto ya poblado atrae de manera explícita a los repobladores, pero en otros casos la fractura histórica del siglo VIII y parte del IX supone una solución de continuidad en la ocupación de dichos lugares.

Como todo sistema de ocupación y control, las líneas defensivas de los castros, claramente estructuradas durante la repoblación tienen una imbricación absoluta con el territorio. Una visión espacial de la localización de las principales líneas establecidas es muy reveladora de la visión del espacio que tuvieron los repobladores.

Hay que señalar que van a ser los valles los elementos fundamentales que atraerán la atención de los nuevos pobladores para controlar el territorio.

En la zona del reino de León la dirección norte-sur de las corrientes fluviales va a ser decisiva en la configuración de la trama urbana. Órbigo, Esla, Cea, Valderaduey, Sequillo, Carrión y Pisuerga van a ser ejes de control y penetración en el territorio. En Castilla, sin embargo, la dirección este-oeste de los cursos determinará el establecimiento de tres barreras paralelas a lo largo del Arlanzón, Arlanza y Esgueva. 
Al sur se configura como gran línea estratégica la propia del Duero, en la que coexistirán los "castra" con "civitates" de mayor empeño, como Zamora, Toro o Simancas.

Hay un tercer factor que es fundamental a la hora de conocer el territorio y su ocupación en la meseta superior, sobre todo en sus zonas centrales y es el contacto campo-páramo que se produce en ellas. En efecto, el páramo y la campiña son dos realidades complementarias que marcan el carácter de los diversos ámbitos. El páramo, boscoso en tiempos medievales, más pobre agrícolamente, determinará una ocupación extensiva muy adecuada para la explotación ganadera. La campiña por el contrario es más apta para el cultivo de cereal y permite una ocupación más intensiva del territorio. Ambas están separadas por las nítidas cortadas, tan características del paisaje de la meseta. No tiene por tanto nada de extraño que sea en estas zonas de contacto, frecuentemente apoyadas en escalones naturales que marcan la diferencia de cota de los dos espacios, donde surjan los puntos de control del territorio desde los que es posible abarcar una gran extensión.

La configuración de los castros era prácticamente homogénea en toda la región. Consistía en un pequeño recinto cercado, donde la función militar y residencial estaban estrechamente imbricadas. Sistemáticamente se asentaban en elevaciones del terreno, más o menos abruptas, con la misión de controlar el territorio. Su envolvente es casi siempre circular o elíptica, adaptándose a la cima del promontorio en el que se asientan. El tipo es absolutamente homogéneo en toda la mitad septentrional de la meseta.

La documentación alto-medieval está repleta de menciones de "villae". Se trata sin duda de la célula residencial básica de asentamiento de la primera repoblación y, aunque las acepciones de "villa" ofrecen diversos matices y no obedecen a una realidad homogénea, hoy conocemos con suficiente aproximación su constitución.

La tipología de "villa" no es homogénea en todo el territorio. En el páramo la estructura de asentamiento consistía en una "hereditas" en torno a un minúsculo núcleo formado por la "corte" con sus casas, herrenes, corrales, etc. Estas fueron de origen familiar, cuyos vínculos continuaron manteniendo los pobladores. La unidad de explotación villa-heréditas se mantuvo durante los tiempos altomedievales, siglo IX-X.

Sin embargo en la campiña se detectan poblaciones más complejas en las que las propiedades se delimitan en las transacciones mucho más detenidamente. Parece que estos núcleos de población estaban ya formados en el siglo $X$ por yuxtaposición de diversas cortes, en las zonas más ricas de la campiña.

Dos son los aspectos que interesa plantear desde el punto de vista de la configuración espacial de estos núcleos. De la documentación se deduce casi invariablemente un carácter cercado o al menos el carácter cercado del espacio denominado "corte" que se puede asimilar al inicial embrión de la villa.

Sea de una forma o de otra, nos encontramos a finales del siglo $X$ con pequeñas "villae" que comprendían varias cortes, cada una de ellas con su cerca y calles o carreras que entre ellas discurrían.

La transformación de estos primeros núcleos, y fundamentalmente las "villae" como célula primaria de ocupación del territorio, en comunidades de aldea o núcleos mayores constituye una de las claves del entendimiento del proceso de la formación urbana de Castilla y León. Aunque es un fenómeno paulatino, y ya se manifiesta durante el siglo $X$, será a lo largo de toda la centuria siguiente cuando se produzca de una manera determinante la cristalización en una red de asentamientos jerarquizada. Varios factores coadyuvarán a este proceso de decantación natural, que desde una estructura indiferenciada llevará a una red mucho menos dispersa y más concentrada de núcleos. La evolución demográfica, en constante progresión durante esos períodos, y el control del suelo, propio del continuo proceso de feudalización que se produce durante este período, son dos aspectos que matizarán y cualificarán este fenómeno de concentración de la pobla- 
ción, que por otra parte se nos aparece como la natural transformación entre un primer asentamiento de carácter más primitivo a una segunda etapa más estructurada y racional desde el punto de vista de la ocupación del territorio.

\section{LA ÉPOCA PLENOMEDIEVAL. | 035-1300. LA CONSOLIDACIÓN DE LA RED URBANA AL NORTE DEL DUERO}

La época en que cristaliza la formación urbana de Castilla y León es aquella que abarca desde la mitad del siglo XI hasta que se consolida dicha red a mediados del siglo XIII. Las tendencias apuntadas a principios del siglo XI de concentración y jerarquización del habitat se acentúan hasta llegar a la eclosión durante el siglo siguiente, que se manifiesta en la aparición de un gran número de núcleos de carácter protourbano, embriones de ciudad y que ya suponen una clara jerarquización y dominio sobre el territorio. Al norte del Duero, esta estructura territorial tendrá un carácter muy distinto según nos refiramos a las zonas centrales de la meseta -los Campos Góticos, los valles bajos del Esla, Carrión, Pisuerga y el propio Duero, junto con los páramos centrales intercalados entre ellos- o a las zonas periféricas del este, norte y oeste de la meseta, en las que los procesos de formación urbana fueron menos intensos.

Al sur, los amplios territorios entre el Duero y el Sistema Central quedan ahora amparados por la posición de Toledo (1085) y se produce una masiva ocupación de los mismos. El sistema utilizado recoge la experiencia que se ha producido durante todo el siglo al norte del Duero, de tal modo que se instituye el sistema de las Comunidades de Villa y Tierra, que permiten ocupar con elevada eficacia grandes territorios con una clara organización territorial y económica.

Al norte los procesos son más complejos. Como norma general podemos afirmar que se produce un fenómeno de desaparición de numerosos núcleos y su concentración en entidades de mayor magnitud, que en numerosas ocasiones constituyen ya un embrión de ciudad.
Aquí es necesario resaltar cómo el proceso de formación urbana se completa con la creciente importancia de algunos núcleos que ya comienzan a descollar durante el siglo XII y comienzos del XIII como verdaderas ciudades, aunque aún es pronto para hablar de tales en el sentido bajo-medieval. Hay núcleos que sobre ese primer escalón de poblaciones de carácter proto-urbano comienzan a despuntar. Algunos de ellos no alcanzarán después el grado de ciudad (como Dueñas, Carrión o Castrojeriz), pero otros, que serán en el futuro las principales ciudades de la región, ya destacan en esta época.

Las cinco ciudades que se consolidarán como sedes episcopales al norte del Duero serán con el tiempo destacados núcleos urbanos. Astorga y León, la última capital del reino, se levantaron sobre los muros de los antiguos establecimientos romanos. Burgos se erigió pronto en el principal núcleo del Condado de Castilla, y potenciado por el Camino de Santiago aparece en el siglo XII como una verdadera ciudad. Zamora verá ampliado durante estos dos siglos (XI y XII) el primitivo recinto que surgió a raíz de la posición conseguida por Alfonso III. Palencia, cuya evolución como núcleo urbano corrió pareja con la fundación del Obispado en 1035, fue una población de señorío episcopal y ya en el siglo XIV era la principal ciudad del entorno, con creciente hegemonía sobre Dueñas, Paredes, Becerril o Monzón.

El proceso general de formación urbana durante dos siglos (XI y XII) transformó radicalmente el sistema de asentamientos en la mitad septentrional de la meseta. Los núcleos que se acaban de citar representan quizá el grado más evolucionado de dicho proceso, junto con algunos matices específicos derivados de su creciente papel institucional. Junto a ellas se desenvuelve el conjunto del proceso, quizá uno de los más sugestivos de la formación urbana de la región, que partiendo de una extensa e indiferenciada red de núcleos rurales (villae, castra) se transforma en un conjunto de asentamientos de carácter protourbano que articulan el territorio. 
Estas villas -no confundir con los núcleos altomedievales- serán núcleos amurallados, contarán generalmente con una organización urbana más compleja, con una estructura pluriparroquial, con la aparición de espacios específicos para las funciones urbanas (plazas o lugares de mercado) y con una clara función de hegemonía territorial. El origen de estos núcleos puede obedecer a primitivos puntos neurálgicos del territorio o a transformaciones posteriores, debidas a una mejor aptitud comercial o agrícola, una vez desaparecida la primitiva función militar.

Falta por señalar brevemente el carácter de la franja septentrional de la meseta desde los confines del Bierzo y el alto valle del Orbigo hasta el páramo de la Lora, que marca la transición al valle del Ebro. Solar de un primer escalón de repoblación durante la segunda mitad del siglo IX, pierde muy tempranamente su papel de avanzada en favor de las zonas centrales de la meseta durante el final del siglo IX y definitivamente durante todo el siglo $X$. Ya hemos comentado como muchos de los repobladores de villas del páramo y de las campiñas durante el siglo $X$ proceden a su vez de núcleos colonizados en las cabeceras de los valles. La pérdida de importancia de estos lugares y su menor aptitud para la agricultura medieval fueron determinando su paulatina decadencia, tanto en la transición hacia el páramo como en las zonas más montañosas de los valles. En estas últimas el hábitat fue siempre más disperso, mientras que ya en la transición hacia la llanura surgió la red de aldeas que conocemos de las otras zonas. Incluso alguna de las posiciones hegemónicas en los primeros momentos fueron cediendo su importancia a localidades situadas más al sur: Cea 0 Amaya son ejemplos de ello, pero también Saldaña, que, aun conservando después el carácter de villa amurallada, perdió su primitivo rango de sede condal frente a la más próspera Carrión, situada aguas abajo del río ya en contacto con las Ilanuras centrales de la meseta, potenciada su importancia por el Monasterio de San Zoilo y por el paso por ella del Camino de Santiago. Este cambio de papel producido entre Saldaña y Carrión en uno de los más importan- tes territorios condales de la meseta es paradigmático del proceso más general producido entre el primer escalón septentrional de repoblación y las zonas centrales consolidadas al alcanzar la línea del Duero.

Si bien el fenómeno de transformación del habitat producido durante el siglo XI y el XII en todas las zonas anteriores fue de gran alcance, mucho más radical fue el producido en las áreas centrales. Se trata de una zona formada por las campiñas y páramos centrales divididos por los valles de los ríos. Como se explicó con anterioridad, nos encontramos ante el paisaje más característico de la meseta: a una cota superior los páramos, formados por calizas pontienses, que componen extensas llanuras cubiertas de monte bajo, dividiendo los amplios valles de sección de artesa invertida, como el bajo Arlanzón, Arlanza, Pisuerga, Carrión, Esla, Duero; y en contraste las extensas campiñas suavemente onduladas y muy aptas para el cereal. Esta zona, de evidentes aptitudes para una economía agrícola medieval, ejerció durante los tiempos medievales un gran poder de atracción, constituyéndose en el centro neurálgico de la meseta. No en vano, tras la división del reino por Alfonso VII (I I57-I230), en los conflictos fronterizos entre sus sucesores fue la zona más disputada y problemática de la frontera.

La zona central, la más próspera de la meseta, a caballo de los dos reinos unificados durante las monarquías de Fernando I, Alfonso VI y Alfonso VII, va adquiriendo una creciente importancia, como lo demuestra la fundación del Obispado de Palencia con un notable señorío y la expansión de la ciudad de Valladolid, que durante el siglo XII se convierte en un próspero asentamiento comercial. Desde la división de los reinos por Alfonso VII en II 57 hasta su definitiva unificación en 1230 por Fernando III, la frontera entre ambos fue teatro de avatares y conflictos, pero sobre todo de un intenso proceso de creación urbana por parte de los monarcas de ambos reinos, aunque más del leonés, cuyo resultado fue una densa red de villas reales en Tierra de Campos y en los principales cauces fluviales leoneses, sobre todo el Esla. 
Sobre esta zona, la densa red de "villae" del siglo $X$ se va transformando durante los siglos $X I$ y Xll en una red de núcleos amurallados 0 cercados que cubren la totalidad del territorio. Sólamente algunos pequeños asentamientos altomedievales permanecen sin cercar durante estos dos siglos. Paralelamente al proceso de concentración y fortalecimiento de todos estos núcleos se produce la paulatina despoblación y consiguiente desaparición de otros núcleos. La aparición de numerosos despoblados documentados durante los siglos XI, XII, XIII son el resultado del proceso de recomposición del hábitat y de la concentración en determinados núcleos.

De los diversos casos conocidos, se deduce que los nuevos pobladores de estos núcleos proceden en gran medida del entorno próximo, esto es, de antiguos vecinos de los núcleos que van quedando despoblados. La entrada en dependencia de una gran parte de los antiguos campesinos libres es un hecho documentalmente comprobado en estas zonas centrales de la meseta. Ello dejaba el campo abierto a los señoríos para trasvasar y concentrar la población en aquellos núcleos que garanticen, mediante un hábitat más estructurado, una más racional ocupación del espacio y un mejor dominio económico del territorio, a la vez que le permiten controlar más eficazmente los movimientos de población. Es muy habitual que el mecanismo normal de población, que queda reflejado en todos los fueros de señorío es la desvinculación de la propiedad del suelo de la de los edificios y la subordinación del valor de los inmuebles al papel desempeñado por los campesinos en la explotación de los bienes raíces productivos. El núcleo urbano queda vinculado a la explotación agraria, en el sentido de que los inmuebles carecen en principio de valor de cambio y sólo son útiles en relación a la residencia del campesinado que cumple una función en el medio agrícola.

Están documentados numerosos casos de villas de fundación real cuyo origen fue un primitivo núcleo altomedieval. Villafrechós, Aguilar de Campos, Bolaños pueden mostrar ejemplos muy significativos. En torno a un pequeño núcleo inicial, surge una ampliación muy supe- rior en extensión que recibe contingentes humanos de pequeños núcleos vecinos, que consiguientemente desaparecerán. En estas ocasiones, la fundación de una villa venía precedida de una intensa actividad de adquisición y preparación de suelo, frecuentemente en terrenos de la iglesia, a la que se compensaba con los beneficios de las futuras parroquias, así como de traslado de pequeños núcleos que desaparecían como tales y llevaban consigo la propia iglesia y su advocación.

Un ejemplo extremadamente esclarecedor lo encontramos en la villa de Paredes de Nava, que durante la Baja Edad Media llegó a ser uno de los principales núcleos de su comarca. En el actual territorio del término se llegan a constatar hasta dieciocho despoblados, casi todos anteriores a la gran crisis del siglo XIV. El paralelismo entre la magnitud de la villa resultante y la cantidad de asentamientos altomedievales luego despoblados es en este caso muy elocuente.

El proceso de transformación de esos iniciales núcleos altomedievales a las villas del siglo XII y XIII como recintos amurallados es uno de los más sugestivos del urbanismo medieval castellano-leonés, y sin duda uno de los más difíciles de desentrañar. Una vez consolidada la "villa" altomedieval se producen procesos espontáneos de ampliación, tanto de yuxtaposición de cortes como de pequeños asentamientos en los accesos al primitivo núcleo. Desde ese primer momento de carácter espontáneo hasta la consolidación de la muralla como eslabón más significativo de una actuación conjunta sobre el núcleo urbano, tienen lugar toda una serie de pasos generados por un trasvase de población propiciado por los señores, laicos o religiosos, en un intento de racionalizar los asentamientos. En este proceso desempeñarán un papel muy importante no sólo el control del suelo y la lotificación en solares para uso privativo o viviendas y el suelo público, para egidos o futuros asentamientos, sino de otros elementos trascendentales en la configuración de estos núcleos, como son las parroquias. Ya veremos más adelante cómo paralelamanete a esta transformación de primitivos núcleos en recintos amurallados se transforman a su vez el carácter de los templos desde su 
concepción dominical y patrimonial en la Alta Edad Media, hasta su papel estructurador en la sociedad que adquieren durante la Plena y Baja Edad Media.

\section{VILLAS FRONTERIZAS}

Las fronteras entre los reinos cristianos tuvieron una cierta trascendencia desde el punto de vista de la repoblación. La necesidad de control de estas zonas determina una fuerte concentración de polos de repoblación en estas áreas para mantener la fidelidad y control de las mismas. Curiosamente las contextura de las distintas fronteras son distintas entre sí. Al sur se encuentra la frontera con el mundo islámico ante el cual no hubo frontera explícita y los procesos de repoblación no fueron aquí significativos. Distinta es la frontera occidental con los reinos de Galicia y Portugal. En esta zona la calidad del terreno era menor y la presencia de algunas villas permitía mantener esta frontera, así Ponferrada, Puebla de Sanabria, Fermoselle o más tarde Ciudad Rodrigo cumplían ese papel. Sin embargo la frontera oriental, con los reinos de Navarra y Aragón, fue siempre teatro de importantes operaciones de creación urbana. En la parte norte, en la actual provincia de Burgos hay numerosos lugares que refuerzan el carácter de zona fronteriza, algunos de ellos con trazado regular como Miranda de Ebro, Medina de Pomar o Briviesca, a los cuales hay que añadir muchos otros como Pancorbo, Santa Gadea, Frías, Oña, Poza de la Sal.

Más al sur se desarrolla la frontera entre los reinos de Castilla y Aragón. Magaña, Ágreda, Ciria, Borobia, Deza, Monteagudo de las Vicarías y Medinaceli cumplían este papel.

Mucha mayor trascendencia desde el punto de vista urbano tuvo la división del reino entre Castilla y León adoptada por Alfonso VII en I I 57 y que duró hasta la reunificación llevada a cabo por Fernando III en 1230. Los dos nuevos reinos reforzaron su línea fronteriza, que discurría por el corazón llano de la meseta. Ambos reinos, ya en los siglos XII y XIII, percibieron con claridad que el mejor medio de reforzar su frontera no era mediante fortificaciones sino fundando villas de pertenencia real. Así, ambos reinos, especialmente el que se sentía más débil, en aquellos momentos el de León, optaron por concentrar las villas en esta frontera. La zona más estratégica de la frontera es la correspondiente a la Tierra de Campos, la zona más rica en aquellos momentos y totalmente llana. Allí los reyes Fernando II y Alfonso IX de León fundan las siguientes villas reales: Benavente, sobre la antigua población de Malgrat, Valderas, Mayorga y Valencia de Don Juan, antiguamente denominada Coyanza, todas ellas en el eje del Esla y Cea. El rey Alfonso VIII de Castilla funda Aguilar de Campos sobre el antiguo lugar de Castro Mayor. Más al norte el rey de León funda Mansilla de las Mulas sobre el Camino de Santiago a su paso por el Esla y más al sur, al pie del Sistema Central, la villa de Miranda del Castañar.

Las villas reales, sobre todo las de Tierra de Campos, componen una tipología de crecimiento específica en la región. Cuando el rey de León decide llevar a cabo la fundación de la villa real ya eran núcleos plenomedievales con la huella de su primer núcleo altomedieval y el recinto posterior con el trazado de su muralla plenamente perceptible en la fotografía aérea. Sobre esta doble presencia se advierte una tercera que corresponde a la fundación real. Todas ellas alcanzan en esta tercera etapa de crecimiento una gran extensión. Valencia de Don Juan alcanza las sesenta hectáreas, Benavente cincuenta y Mayorga y Valderas más de veinte cada una.

\section{LAS POBLACIONES DE LA EXTREMADURA}

En el norte del Duero observamos un proceso de decantación de los sistemas productivos y de asentamientos, en tanto que en el sur se produce un esfuerzo de planificación que recoge la experiencia acumulada en la génesis del sistema urbano de los territorios anteriores. El sistema empleado es el de las Comunidades de Villa y Tierra. La villa se convierte en un lugar fuertemente protegido $y$ favorecido desde el punto de vista jurídico, directamente ordenado bajo la actuación real 
y donde en los primeros momentos surge una pujante estructura comunal. Y a su vez se convierte en cabeza de un extenso territorio de carácter fronterizo.

Este sistema se mostró tremendamente eficaz de cara a la repoblación y control del territorio, ya que con un reducido número de centros de poder permitía a los monarcas un efectivo control del territorio. Por otro lado la creación de fuertes concejos, que no tenían dependencia señorial, permitía reforzar el poder de la corona.

En la Extremadura -actuales provincias de Soria, Segovia, Ávila y Salamanca-, todo el papel urbano se concentra en las cabezas de comunidad, que atesoran todas las funciones atribuidas a las ciudades medievales. En efecto, fuera de ellas nos encontramos un gran número de aldeas, ninguna de ellas cercada y que jurídica e institucionalmente aparecen subordinadas a la cabecera.

A cada una de estas ciudades cabeceras de comunidad corresponden diversas aldeas y todo el territorio que a estas corresponde. Varía este número entre las diversas comunidades, pero las mayores Ávila y Segovia tienen más de cien aldeas, no sólo en la Extremadura sino también en la Transierra. Las aldeas de la comunidad carecían de un elemento tan urbano como es la muralla y tienen una sola parroquia.

Las características que definen a las villas que se están formando al norte del Duero, aparecen como punto de partida en la formación de las cabezas de la comunidad: la estructura de parroquias y la muralla son los aspectos físicos más evidentes en todas ellas, pero en su base se encuentran una cierta estructura institucional, unos mecanismos de control del suelo que posibilitan el desarrollo del núcleo urbano, una ordenación jurídica mediante fueros o cartas pueblas, y una creciente complejidad funcional. En la Extremadura estos aspectos se dan de manera mucho más clara. La iniciativa de su formación procede casi siempre de la Corona y el concejo aparece desde los primeros momentos organizando la vida de la comunidad, mientras que la influencia de los fueros, muy ventajosos para atraer pobladores, es decisiva en su formación.

Además de esta primera característica diferencial de los núcleos de la Extremadura, consistente en una explícita voluntad de creación urbana, hay otro aspecto definidor de estos núcleos derivado asimismo de su papel territorial. Comparado con los núcleos al norte del Duero, las villas y ciudades de la Extremadura castellana y leonesa tienen por término medio una mayor magnitud y complejidad en su estructura urbana. Dejando al margen Salamanca, Segovia y Ávila, que son los mayores concejos de la Extremadura, a los que habría que añadir su condición de sede episcopal, muchos de estos núcleos alcanzan por su población, superficie urbana y número de parroquias una mayor relevancia que la media de los núcleos del norte. Sólo las históricas ciudades institucionales como Zamora, Toro, Burgos, León, y algunas villas reales fronterizas de Tierra de Campos (Mayorga, Benavente, Villalpando, Valencia de Dom Juan) les son comparables. En efecto, Soria, Salamanca, Segovia, Ávila, Cuéllar, Olmedo y Medina superaban las quince parroquias y otras mucho menores, como Maderuelo o Calatañazor tuvieron nueve.

Las características, por tanto, de las villas y ciudades de la Extremadura se pueden resumir como sigue: son cabezas de una Comunidad de Villa y Tierra, con lo que se erigen en centros de un territorio subordinado; su magnitud y superficie media es mucho mayor que las villas de la mitad septentrional de la meseta; su status jurídico e institucional más estructurado y organizado, siendo además de gran homogeneidad a lo largo de todo el área; su génesis y formación aparece planificada a partir de su carta de población, aunque en ningún caso se base en un plano regulador; la estructura física es más compleja, con mayor número de parroquias y más diversificada en sus espacios urbanos. Esta estructura nuclear o de colaciones viene potenciada por la diversa procedencia de los pobladores, que se agrupan en torno a una parroquia y que es mucho más heterogénea que en los núcleos del norte, que casi siempre se nutren de los núcleos de su entorno. Al partir de un acto de voluntad más 
explícito, los límites del asentamiento, esto es la muralla, suele venir prefijada al inicio de la repoblación. Debido a la función militar de las Comunidades y a la necesidad de poder dar cobijo a los ganados en tiempos de guerra, algunas murallas engloban más suelo del necesario para la población (Salamanca, Soria y Sepúlveda son los ejemplos más significativos).

\section{EVOLUCIÓN URBANA Y TERRITORIALIZACIÓN ECLESIÁSTICA}

La comparación entre las dos etapas de la estructura religiosa que corresponden a las dos fases de la evolución urbana -altomedieval y pleno o bajomedieval- muestra los cambios producidos en ella por la reforma gregoriana y por la introducción de la influencia cluniacense, precedida y potenciada por los diferentes concilios que tuvieron lugar durante los siglos XI y XII, alguno de ellos en León y Castilla.

La primera época nos muestra a la iglesia profundamente enraizada en los procesos de feudalización. Precisamente algunos de los más importantes, y sin duda mejor conocidos, dominios feudales de la meseta son los de los grandes monasterios del norte del Duero: San Pedro de Montes, San Isidoro de León, San Facundo y San Primitivo, San Pedro Cárdeña, San Pedro de Arlanza o San Millán de la Cogolla. Nos ha sido perfectamente transmitido, gracias a las fuentes documentales de los mismos cómo estos monasterios fueron constituyendo sus dominios monásticos según una gama de compras, donaciones y entradas en dependencia, que nos muestra todo un variado repertorio de modos de penetración del poder feudal en una primitiva sociedad de presores. Las primeras diócesis -León, Astorga, Zamora- estaban sin duda más cercanas a un modelo de señorío, similar al de los grandes cenobios, que a una estructura institucional.

Finalmente, la existencia de iglesias y santuarios propios, en los que el señor o propietarios mantenían el derecho de presentación de clérigos, entra de lleno en el concepto patri- monial de los lugares eclesiásticos coherente con una sociedad feudal.

Los cambios producidos por la reforma gregoriana, potenciados en León y Castilla a partir del reinado de Alfonso VI por la influencia cluniacense, supusieron un cambio en la posición de la iglesia.

Si bien, explícitamente, la reforma gregoriana aboga por un distanciamiento de los poderes temporales y un reforzamiento de la estructura institucional, ello coincide sin duda con la nueva realidad de la vida urbana, que surgía junto al modo de producción feudal como un campo abierto con un elevado potencial de estructuración social. La iglesia adecua su estructura secular a esta nueva realidad, alejándose de su anterior configuración patrimonial. De este modo potencia la creación de la parroquia como célula religiosa básica, pero también administrativa y fiscal, desgajándola de la antigua dependencia dominical de las primitivas "iglesias propias". Ello produce un reforzamiento de la estructura eclesiástica y, sobre todo, del papel de la parroquia, la cual, identificada con la aldea en lo rural y con la colación o barrio en lo urbano, será la célula básica de la organización urbana.

La realidad de la parroquia se va fraguando desde finales del siglo XI y se consolida durante todo el siglo posterior. En las más tardías poblaciones y en las ciudades de la Extremadura la composición parroquial es más compleja; ello obedece a que cuando esos núcleos surgen ya está formulado el concepto de parroquia y ésta aparece como la célula básica de la nueva estructura urbana.

\section{LA CIUDAD MEDIEVAL DE CASTILLA Y LEÓN}

Una serie de aspectos específicos caracterizan a la ciudad de esta región en los tiempos medievales, sobre todo en la relación con el paralelo fenómeno de formación urbana que en toda Europa Occidental se produjo en aquella época. 
El rasgo más definitorio es, sin duda, el derivado de la gran cesura histórica que supuso la invasión musulmana. Debido a ello, la herencia urbana tardo-romana, que en otros lugares de Europa resultó significativa, desaparece casi por completo en la región del Duero. Sólo dos ciudades fueron reconstruidas sobre la base de establecimientos y trazados romanos, León y Astorga; sólo ellas fueron de algún modo herederas de la tradición clásica. Pero aún así, sólo la muralla aparece como un potente antecedente urbano, pero no así el trazado, que se transformó radicalmente. El resto de poblaciones que se reinstauraron sobre antiguas ciudades romanas, fue sólo a efectos de recuperación del asentamiento, de modo que no se encuentran vínculos de conexión con el antiguo trazado.

En lo referente a la herencia de las ciudades del pasado, tiene un peso importante el hecho de que toda la red urbana sea establecida de nuevo. El término "repoblación" se traduce en la historia urbanística en términos de instauración urbana. La práctica totalidad de los núcleos surgen de nuevo a partir del siglo IX, sin antecedentes importantes que incidan en su morfología.

Este período, que ve surgir acompasadamente los núcleos urbanos en otras regiones de Europa, se reduce en nuestra región -y en los demás reinos cristianos peninsulares- a un estrecho margen, que transcurre entre finales del siglo IX y comienzos del XIII. En este pequeño lapso de tiempo, la mitad septentrional de la meseta se puebla de una multitud de organismos preurbanos. Esta floración, que surge en un primer momento con un carácter de libertad, de individualidad y de dispersión, se organiza y se jerarquiza a una velocidad inusitada, apareciendo los procesos de intensa feudalización que se estaban produciendo en el resto de Europa y que precisamente ya se habían comenzado a generalizar en la época visigótica. A finales del siglo $X$ y comienzos del siguiente la feudalización era ya un fenómeno plenamente desarrollado. En poco más de un siglo, una primera hornada de pioneros, con un derecho embrionario y una organización sumaria, había dado paso a una sociedad mucho más evolucionada, coherente con la perspectiva de la época.

El siglo XI fue muy intenso en cambios estructurales en el sistema urbano. La concentración del hábitat y su jerarquización fueron el resultado lógico de un proceso de decantación de las estructuras productivas y demográficas. A un período inicial de dispersión, sucede otro de concentración y racionalización del sistema de asentamientos. Sin duda esto fue potenciado por el creciente poder feudal para mejorar sus rendimientos y el control sobre sus dominios. En dicho siglo se produce el paso de los asentamientos altomedievales a los núcleos plenomedievales -las aldeas y las villas amuralladas-.

El siglo XII, siglo de esplendor urbano en la Europa occidental, ve consolidarse en la región las estructuras urbanas que acaban de surgir en la época precedente, así como aparecer la nueva hornada de villas y ciudades de la Extremadura. Es muy difícil distinguir los crecimientos de uno y otro siglo, y se trata en conjunto como una sola etapa, si bien en ella se producen dos fenómenos que en otros lugares de Europa son sucesivos en el tiempo: la adquisición de un grado urbano y su propio proceso de crecimiento.

Como demuestran las fuentes demográficas y la propia evolución de las ciudades, el crecimiento urbano sufre una ralentización a partir del segundo cuarto del siglo XIII. Esta última etapa de florecimiento de las grandes ciudades medievales europeas, donde surgen los terceros y cuartos recintos, ya con superficies muy superiores a las cien hectáreas, y donde se reagrupan en una sola cerca los diversos burgos de cada ciudad, está casi ausente en nuestra región.

Esta compresión, en tres siglos, de un proceso que en otros lugares de Europa se desarrolló durante el doble período de tiempo, tuvo unas indudables consecuencias en la estructura de la red urbana. A ello hay que se que se trata de fenómenos de creación urbana en unos territorios vírgenes en cuanto a organi- 
zación territorial. Ello supuso que se multiplicaran las actuaciones de localización, según distintas pautas y factores que se superponen en el tiempo. Así, a modo de ejemplo, constatamos en el siglo XII al menos cuatro fenómenos simultáneos de génesis urbana de distinta etiología. En primer lugar, la propia evolución de los núcleos protourbanos, que paulatinamente se transforman en urbanos, reciben sus fueros o carta de población, se dotan de su muralla y alcanzan una estructura parroquial que les articula. Al mismo tiempo, se realiza todo el esfuerzo de implantación de las Comunida- des de Villa y Tierra al sur del Duero, con todo lo que ello supone de intensos procesos de creación urbana. Por otro lado, se refuerzan las fronteras entre reinos cristianos, utilizadas por los monarcas para incrementar su control en el juego de fuerzas medieval, y su paralelo fenómeno de génesis de organismos urbanos. $Y$, finalmente, se comienzan a producir los primeros intentos de implantaciones urbanas para articular la colonización de algunas zonas marginales. Todo ello nos da idea de la gran cantidad y variedad de núcleos urbanos que se generan simultáneamente en la región. 


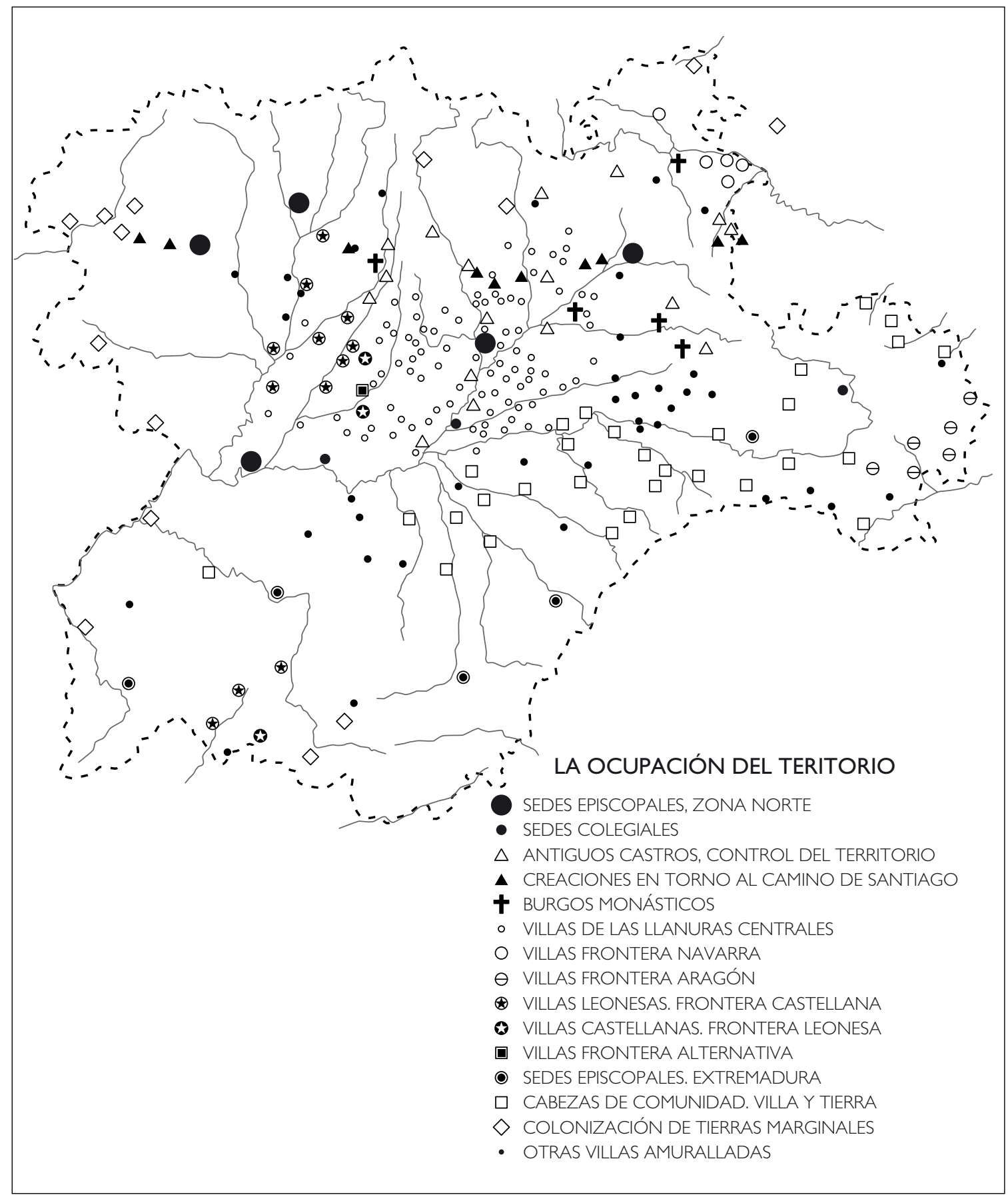

Fig. I. La ocupación del territorio 


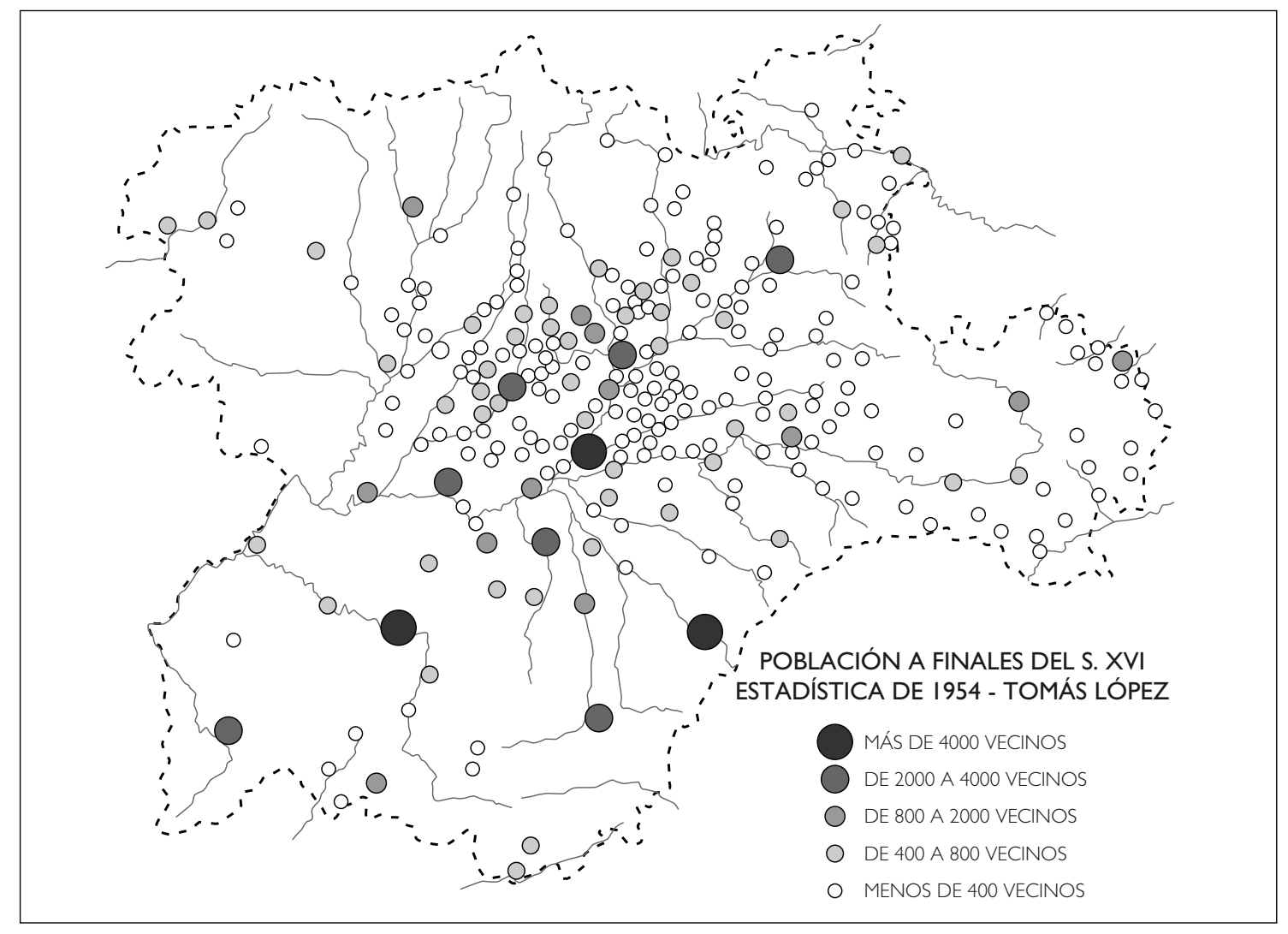

Fig. 2. La población a finales del S.XVI

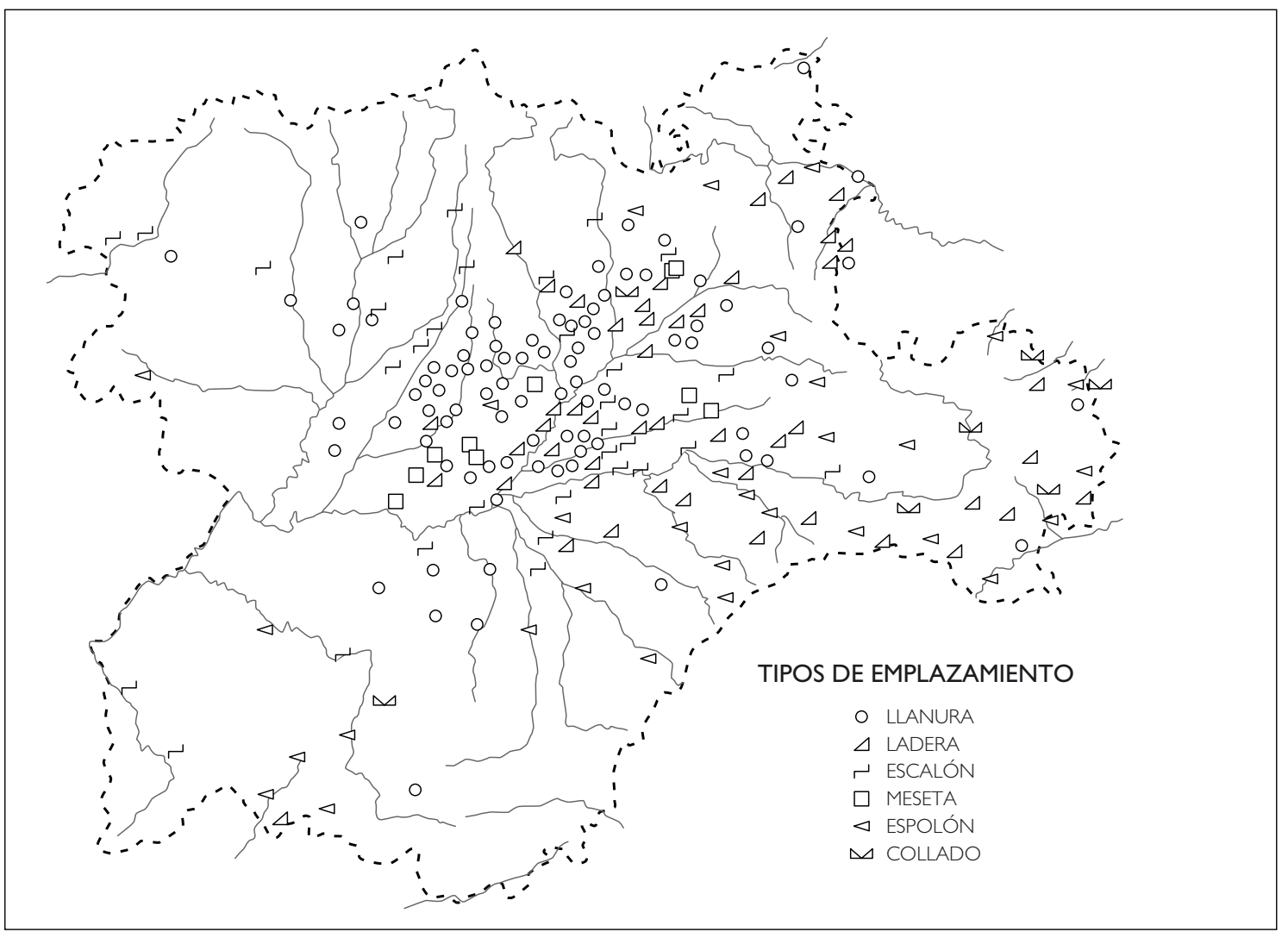

Fig. 3. Tipos de emplazamiento 


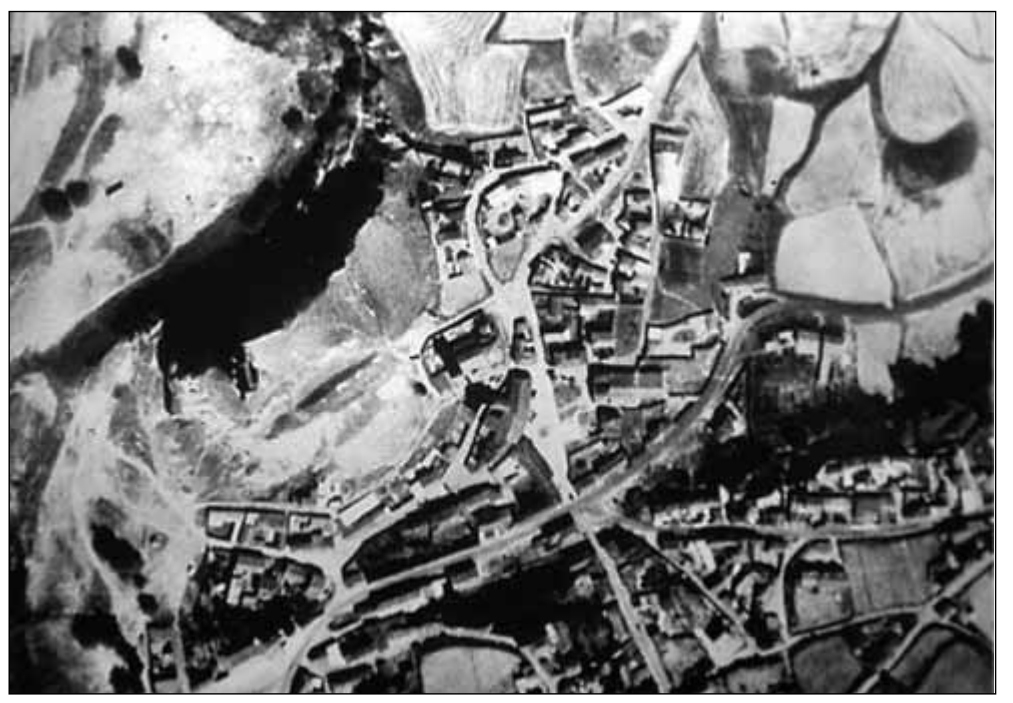

Lám. I. Cea. Esta villa en la cabecera del Cea tuvo gran relevancia en la alta Edad Media, de lo cual quedan testimonios. En la fotografía aérea se aprecia la considerable dimensión de este primer recinto, situado al noroeste del núcleo y en la actualidad deshabitado, salvo la iglesia.

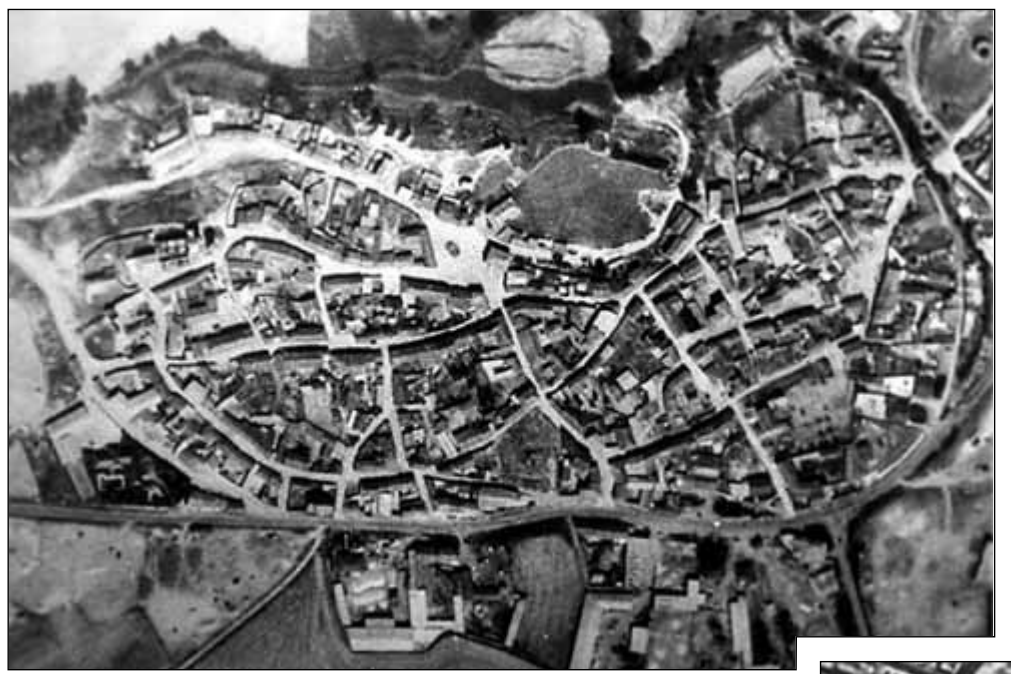

Lám. 2. Melgar de Arriba. En esta población del río Cea se observa muy bien la existencia de un pequeño recinto altomedieval, un castro de apenas una hectárea, junto al paso del río, que queda englobado en el recinto plenomedieval, mucho mayor.

Lám. 3. León. La capital del reino heredó el recinto rectangular romano, sobre el que superpuso un viario propiamente medieval de conexión entre puertas. A pesar de los dos arrabales medievales el conjunto de su superficie no es extenso al contrario que ocurre con las fundaciones más meridionales.

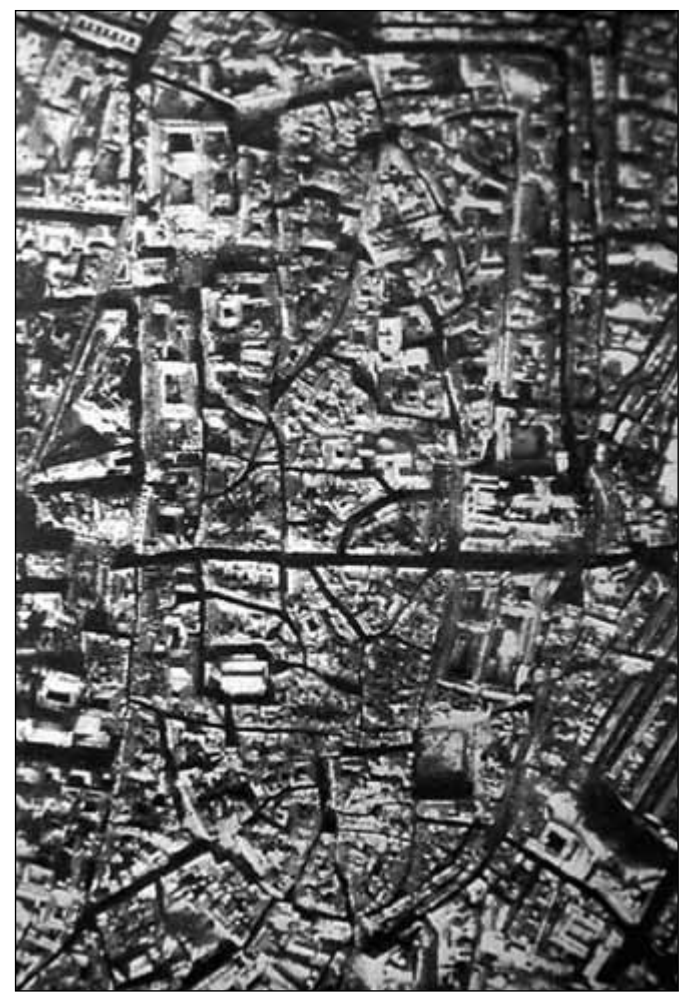


Lám. 4. Zamora. Esta ciudad, posición avanzada sobre el Duero del reino de León y fundación episcopal desde su origen, muestra uno de los conjuntos urbanos más complejos de la región. En él se advierten: un recinto altomedieval de cierta entidad en el extremo occidental del espolón; un recinto plenomedieva del siglo Xl; una fundación de Alfonso VI contemporánea de las de las extremaduras, una puebla cercada en el valle de fundación tardía $y$, finalmente un completo conjunto de arrabales.

Lám. 5. Valladolid. En el centro de la meseta esta población, sustentada por una colegiata, acabó teniendo en la Baja Edad Media el mayor recinto en superficie de la región. Al interior de esta última cerca de mas de cien hectáreas se pueden observar el recinto del siglo XI y otro debido a la ampliación en función de la colegiata.

Lám. 6. Miranda del Castañar. Esta villa salmantina, situada ya en la vertiente sur de la cordillera, fue fundada a principios del siglo XIII por Alfonso IX, lo cual se advierte en su trazado con todas las calles de la mitad norte, paralelas entre sí, perpendiculares a la calle Derecha y descendiendo por la máxima pendiente.
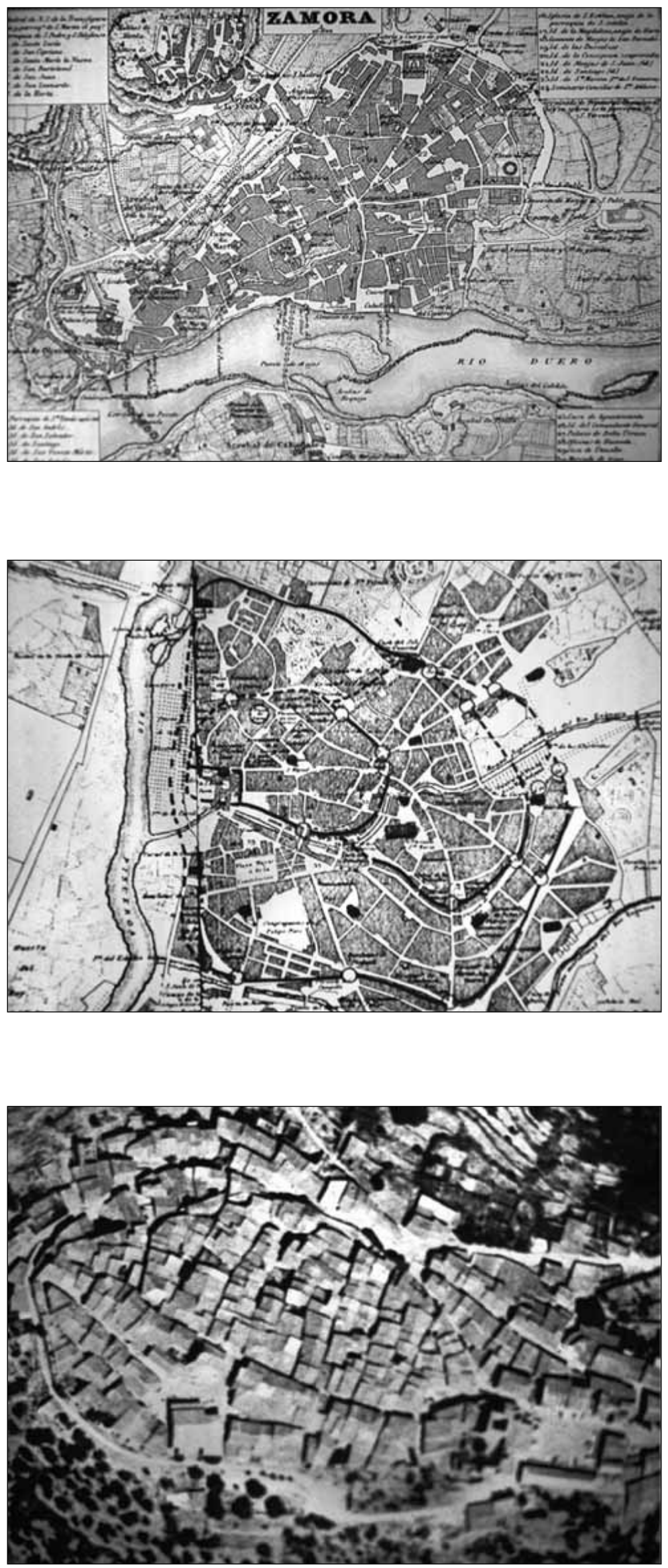


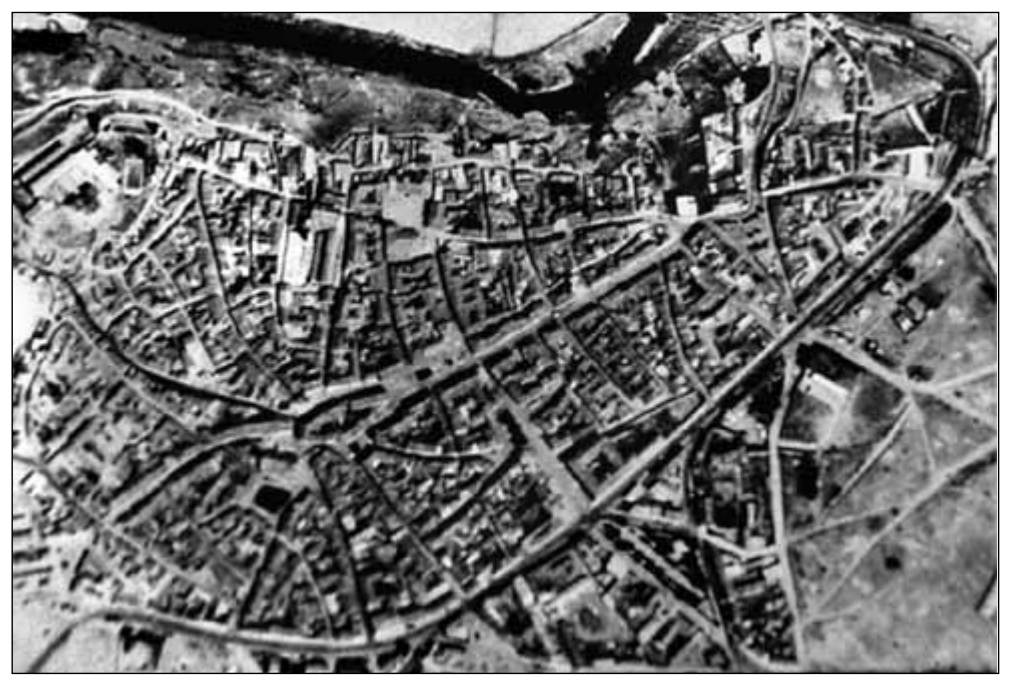

Lám. 7. Valderas. Característica villa real leonesa en la frontera con Castilla. Se advierten los tres recintos: al noroeste el antiguo castro altomedieval, junto al río Cea. Hacia el este se percibe con claridad el recinto plenomedieval, bastante mayor. Finalmente vemos como la extensión del núcleo se duplica hacia el sur con la fundación real

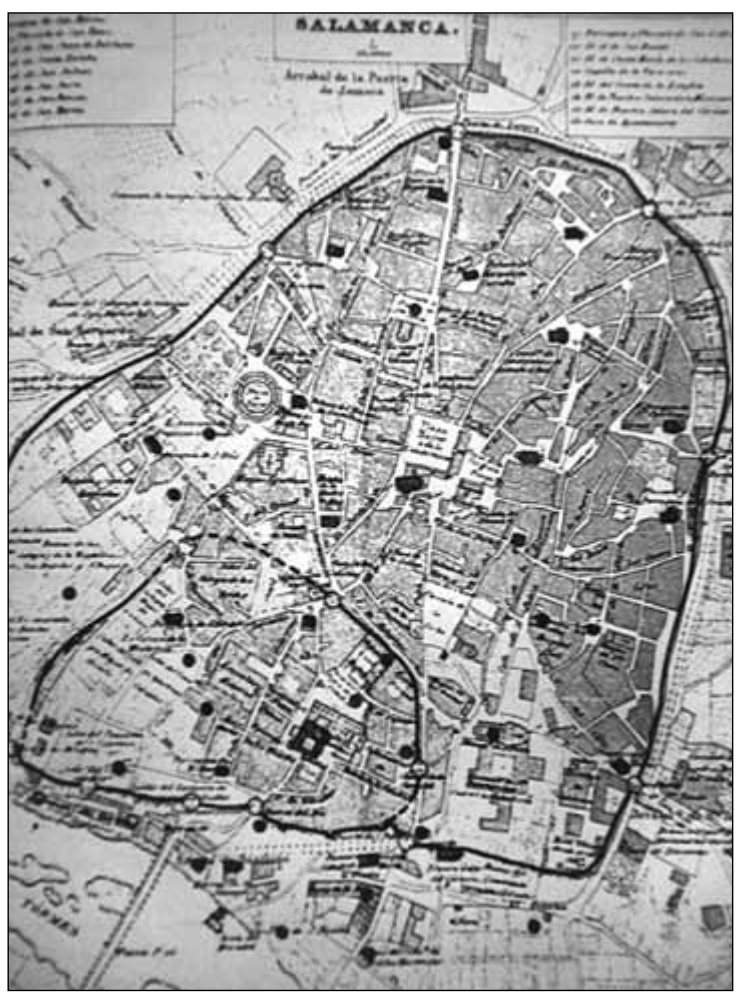

Lám. 8. Salamanca. Típica población de la Extremadura, con un primer recinto y otro mayor de casi cien hectáreas que engloba al primero y con muchas parroquias como mecanismo de repoblación, casi todas ellas intramuros, debido a la holgura del recinto. 
Lám. 9. Soria. Que esta ciudad es cabeza de comunidad de villa y tierra queda claro por su configuración urbana: recinto muy extenso, que la ciudad no alcanzó a colmatar y gran número de parroquias con las pequeñas colaciones alrededor de ellas. Queda también muy clara en la imagen la función de la ciudad como control del paso del Duero entre Castilla y Aragón.

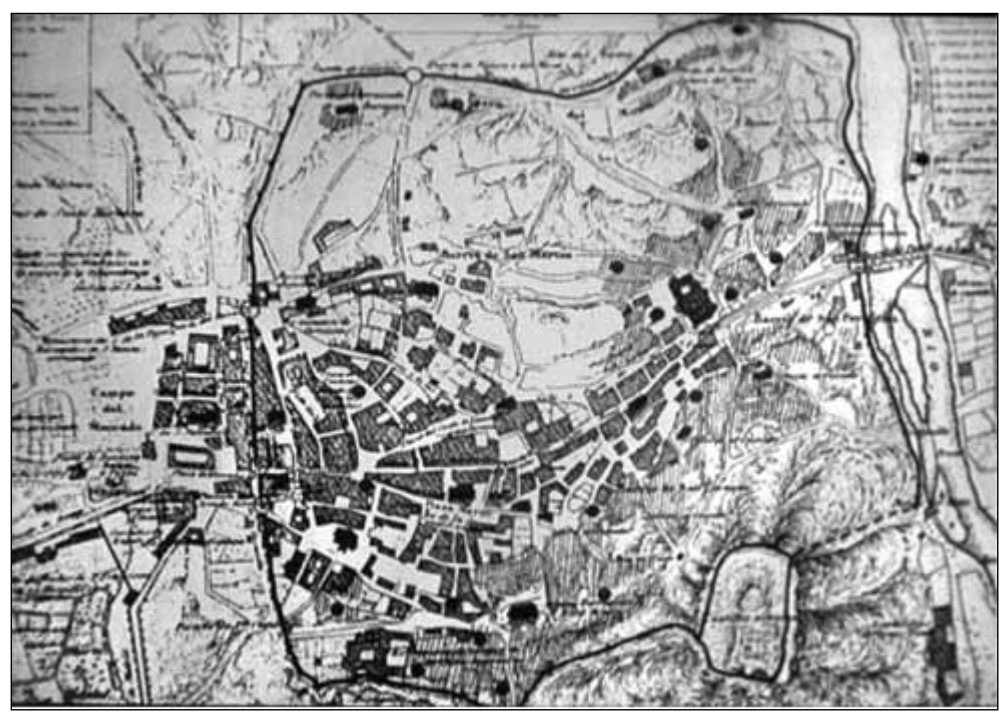

Lám. 10. Burgo de Osma. Cuando se planteo la restauración de la sede episcopal en los teritorios orientales de la Extremadura no se optó por la principal ciudad, Soria, sino por situarse junto a la antigua sede visigótica Uxama. Al pie de las ruinas de la antigua ciudad se fundó el Burgo de Osma, mitad conjunto episcopal, de las más completos de Castilla, mitad ciudad regular con calles paralelas.

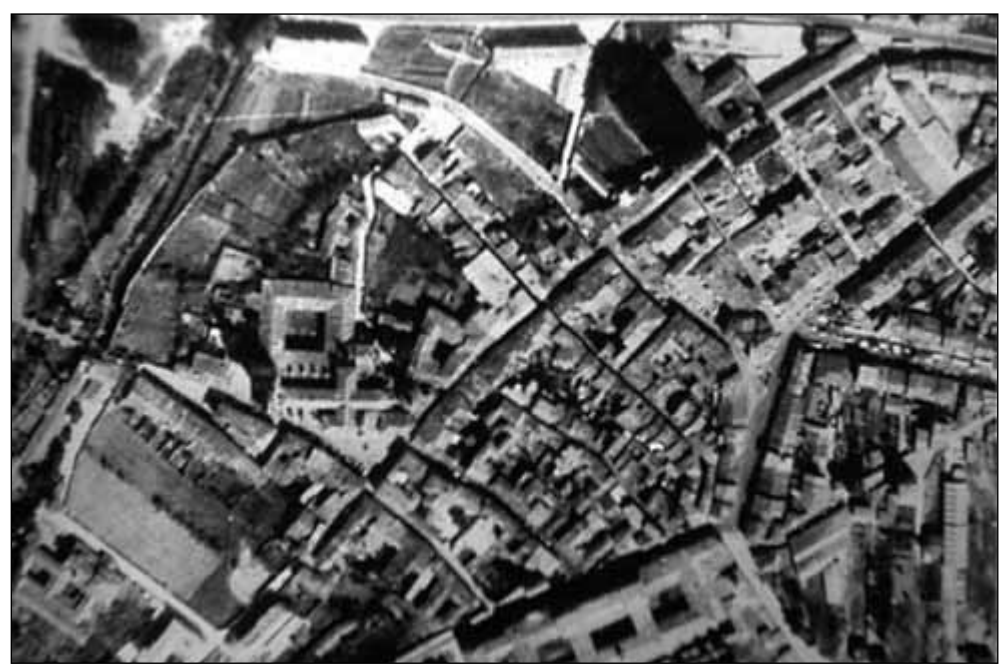

